Mota, N. B., Copelli, M., \& Ribeiro, S. (2016). Computational tracking of mental health in youth: Latin American contributions to a low-cost and effective solution for early psychiatric diagnosis. In D. D. Preiss (Ed.), Child and adolescent development in Latin America. New Directions for Child and Adolescent Development, 152, 59-69.

\title{
Computational Tracking of Mental Health in Youth: Latin American Contributions to a Low-Cost and Effective Solution for Early Psychiatric Diagnosis
}

\author{
Natália Bezerra Mota, Mauro Copelli, Sidarta Ribeiro
}

\begin{abstract}
The early onset of mental disorders can lead to serious cognitive damage, and timely interventions are needed in order to prevent them. In patients of low socioeconomic status, as is common in Latin America, it can be hard to identify children at risk. Here, we briefly introduce the problem by reviewing the scarce epidemiological data from Latin America regarding the onset of mental disorders, and discussing the difficulties associated with early diagnosis. Then we present computational psychiatry, a new field to which we and other Latin American researchers have contributed methods particularly relevant for the quantitative investigation of psychopathologies manifested during childhood. We focus on new technologies that help to identify mental disease and provide prodromal evaluation, so as to promote early differential diagnosis and intervention. To conclude, we discuss the application of these methods to clinical and educational practice. A comprehensive and quantitative characterization of verbal behavior in children, from hospitals and laboratories to homes and schools, may lead to more effective pedagogical and medical intervention. (๖) 2016 Wiley Periodicals, Inc.
\end{abstract}


$\mathrm{M}$ ental suffering during childhood is a serious concern, hard to diagnose and manage, and prone to have neurodevelopmental impacts. A mentally or emotionally impaired child often fails to learn school content or to develop proper social skills, and the persistence of these symptoms greatly hinders one's life course. Since their original psychiatric description, the early signs of mental disorders such as schizophrenia are associated with clinical severity (Kessler et al., 2007; Kessler, Keller, \& Wittchen, 2001), symptom persistence (Clark, Jones, Wood, \& Cornelius, 2006; Kessler et al., 2007), and lack of response to treatment (Kessler et al., 2007; Nierenberg, Quitkin, Kremer, Keller, \& Thase, 2004). Symptoms that go unrecognized can contribute to the appearance of depression, low selfesteem, chronicity, school absenteeism, social isolation, and risky behavior (Kessler et al., 2007; Oschilewsky, Gomez, \& Belfort, 2010). In order to prevent major impacts, it is thus necessary to identify the psychiatric risk with precision and as early as possible.

A review of the prevalence of mental disorders in youth reveals a very wide variation across different studies. For instance, the prevalence of mental suffering in childhood and adolescence over the past four decades ranges from $1 \%$ to $51 \%$, depending on the publication chosen (Fleitlich \& Goodman, 2000; Roberts, Attkisson, \& Rosenblatt, 1998). This major variability is likely due to inconsistencies in the instruments used to screen the pathologies, in the severity of symptoms, and in the source of information. The psychiatric evaluation of children poses a major challenge because it is difficult to obtain reliable reports of internally generated symptoms. Indeed, to characterize mental symptoms in children, it is necessary to also interview other sources, such as parents, other relatives, and teachers. It is also critical to ensure that the child patient clearly understands the questions posed. Some concepts are not easy to explain, and cultural differences regarding what is considered a pathological behavior often impair the child's ability to communicate. For example, interviews with teachers tend to reveal a higher prevalence of hyperactivity in children than do interviews with parents (Fleitlich \& Goodman, 2000).

When criteria applied in developed countries were applied in developing countries, higher prevalence has often been found (Fleitlich \& Goodman, 2000; Roberts et al., 1998). In Great Britain, the overall prevalence of mental disorders during childhood is substantial, reaching 9.5\% (de la Barra, 2009; Ford, Goodman, \& Meltzer, 2003; Oschilewsky et al., 2010). In Latin America, studies report a large variability of prevalence. A study conducted in Chile found an overall prevalence of $22.5 \%$ for ages 4 to 18 (de la Barra, Vicente, Saldivia, \& Melipillán, 2012). A review including Latin American countries reported prevalence of psychiatric disorders during childhood ranging from $5 \%$ to $22 \%$, a large variance that is explained by methodological differences across studies (de la Barra, 2009). One example is a study performed in Puerto Rico, age range 4 to 17 years old, which found a prevalence of mental disorders of $19.8 \%$ when considering 
Diagnostic and Statistical Manual of Mental Disorders-IV criteria with or without impairment, but the prevalence decays to $16.4 \%$ when only impairment cases were considered, and to $6.9 \%$ if a measure of global impairment was added (Canino et al., 2004; de la Barra, 2009). A multicentric study in developing countries of Africa, Asia, and South America revealed a prevalence of mental disorders ranging from $12 \%$ to $29 \%$ for ages 5 to 15 , with higher prevalence in South American countries (Fleitlich \& Goodman, 2000; Giel et al., 1981).

In developing countries, poverty and social development are key factors affecting mental health. In Latin America, mental disorders are significantly related to social vulnerability during childhood, such as homelessness or dropout from school (Belfer $\&$ Rohde, 2005; Oschilewsky et al., 2010; Rohde, Celia, \& Berganza, 2004). The causal link between social vulnerability and mental disorder changes direction across different diagnostic entities. For schizophrenia there is evidence pointing to social selection, i.e., the development of symptoms leads to social impairment. In contrast, for depression, antisocial personality and substance abuse, the evidence points to social causation (Dohrenwend et al., 1992; Robins \& Price, 1991). In all cases, prevention and early differential diagnosis are likely to help the patient manage a difficult situation, but large-scale interventions over entire populations need to be properly designed in order to have real social impact.

We need to understand the mental health epidemiology of Latin American children, so as to overcome the lack of information about this issue globally (Baxter, Patton, Scott, Degenhardt, \& Whiteford, 2013), and even the lack of information about general mental health epidemiology in Latin America (Baxter et al., 2013; Duarte et al., 2003; Mercadante, Evans-Lacko, \& Paula, 2009; Oschilewsky et al., 2010). Studies of this topic used a variety of different methods to search for symptoms and diagnosis (different instruments and settings) (Duarte et al., 2003), and yet found results similar to those found in developed countries, but with more prevalence of risk factors like poverty, parental mental disorders, and family violence (Duarte et al., 2003). Given the high poverty rate and low educational level in the region, it is likely that there is in Latin America an undiagnosed population undergoing mental suffering without access to proper diagnosis and treatment, because of the expensive and ineffective diagnostic models used in most of these countries.

In order to stop this vicious cycle of mental suffering and social impacts, especially important in developing countries like in Latin America, we will need to be creative. There is great hope in the interdisciplinary field of computational psychiatry. Here, we review the advances on this new field, focusing on automated diagnosis tools for psychiatric diseases. We also present quantitative speech measurements adequate for large-scale analysis and able to improve the recognition of pathological and nonpathological neurodevelopmental paths within clinical and educational settings. 


\section{Computational Psychiatry: New Methods for Understanding Human Behavior}

For over a century, psychiatry has described the psychopathology of diagnostic entities as patterns of deviant behaviors. The diagnostic manuals (First, Spitzer, Gibbon, \& Williams, 1990) emerged as a consensus among experts, stating which associated symptoms should be considered as a diagnostic entity, for how long and under which circumstances (Krystal \& State, 2014). However, after decades of hard effort and bulky scientific investment, the known biomarkers are not specific for any psychiatric symptom-based diagnosis, because behavioral symptoms are multidetermined (Insel, 2014).

A distinct approach based on transdiagnostic dimensions has recently emerged in psychiatry. The Research Domain Criteria (RDoC; Insel, 2014; Insel et al., 2010; Kaufman, Gelernter, Hudziak, Tyrka, \& Coplan, 2015) classify population samples by grouping similar disorders within certain domains of behavior. This strategy has been particularly interesting for child psychiatry, because it allows a better assessment of the risks associated with abuse experienced by vulnerable infants (Kaufman et al., 2015). The search for better diagnostic strategies is an essential part of the effort to break the cyclic link between mental disorders and social damage. In that regard, childhood represents an early window of opportunity for the identification of cognitive deficits and mental disorder. The hope is that adequate timely intervention may revert poor prognoses and establish interventions able to effectively minimize damage to the individual and his or her surroundings.

The correlation of behavior with biomarkers can be meaningful only if the quantitative measurements are both comprehensive and precise. The nascent field of computational psychiatry employs increasingly sophisticated mathematical tools to precisely quantify behavior, so as to better grasp the relationship between biological variables (genetic, biochemical, neural) and purely behavioral variables such as performance on cognitive tasks or psychometric scales (Adams, Huys, \& Roiser, 2015; Montague, Dolan, Friston, \& Dayan, 2012; Wang \& Krystal, 2014).

Even when such a relationship cannot be clearly established, it is possible to search for clusters within the population based on the variability of the biological (Brodersen et al., 2014; Wang \& Krystal, 2014) or behavioral (Bedi et al., 2015; Bertola et al., 2014; Cabana, Valle-Lisboa, Elvevag, \& Mizraji, 2011; Díaz, 2013; Elvevag, Foltz, Weinberger, \& Goldberg, 2007; Montague et al., 2012; Mota, Furtado, Maia, Copelli, \& Ribeiro, 2014; Mota et al., 2012; Yoshida et al., 2010) data measured. The hope is that the symptomatic characterization of each cluster will greatly advance the understanding of the psychopathological mechanisms underlying a wide variety of mental disorders. This knowledge may not only help the early identification of individuals suffering from mental disorders but may also contribute to the design of low-cost yet effective interventional methods able to prevent 
major cognitive deficits and their consequences, with potential to improve the psychiatric scenario in Latin American countries.

\section{Automated Diagnostic Tools: Hope for Early Intervention}

Computational psychiatry is still a young discipline, but there are already some identifiable advances in the development of tools capable of quantifying core behaviors affected in mental disorders. In the past 5 years, researchers from Uruguay, Brazil, and Argentina pioneered the development of computational tools for the automatic analysis of psychopathological speech; these innovative tools yield very good diagnostic performance (Bedi et al., 2015; Bertola et al., 2014; Cabana et al., 2011; Mota et al., 2012, 2014), and even predict psychiatric outcomes in the prodromal phase (Bedi et al., 2015). Application of these techniques to Latin American samples demonstrated the feasibility and advantages of these methods in developing countries (Bertola et al., 2014; Mota et al., 2012, 2014).

Language can be understood as a window into the organization of thoughts and therefore able to reflect fundamental aspects of mental functioning. Through speech we present to others what and how we think and feel, allowing the establishment of social bonds. Language features such as the structure of the trajectory of words (Bertola et al., 2014; Mota et al., 2012, 2014; Wang \& Krystal, 2014), semantic consistency (Bedi et al., 2015; Elvevag et al., 2007), and prosody (Grunerbl et al., 2015) can automatically be measured to characterize psychopathological aspects of different mental disorders.

With regard to child psychiatry, the early identification of chronic developmental disorders such as autism is in order. It is known, for example, that patients within the autism spectrum have a peculiar way of interacting with toys, a behavior highly amenable to accurate automatic measurements (Westeyn et al., 2012). Also common in the autism spectrum are deficits in the ability known as theory of mind, which is involved, for instance, in the capacity to understand that the beliefs of others may differ from one's own beliefs (Baron-Cohen, Leslie, \& Frith, 1985; Frith, 1997; Misra, 2014). In a game designed to investigate theory of mind in autistic patients, participants are rewarded for choosing a cooperation strategy that requires one to understand that other participants have ideas different from his/her own. When playing this game, people diagnosed within the autistic spectrum rely significantly less on the cooperation strategy that requires theory of mind, in comparison with control participants. Importantly, this measure of cooperation correlates with the severity of the symptoms (Yoshida et al., 2010). This is a compelling example of how an elusive, hard-to-measure behavioral skill can now be accurately quantified in a substantially less biased manner, generating a stream of objective data as the experimental subject behaves freely. 
Also important for child psychiatry is the early onset of psychotic disorders. One of the main symptoms of psychotic illnesses are disorders of thought (Andreasen \& Grove, 1986), characterized by a fuzzy sequence of words produced in spontaneous speech, with a higher rate of unusual associations than in the general population (Bleuler, 1911; Kraepelin, 1906; Moskowitz \& Heim, 2011). Thus, it is to be expected that the consecutive combination of words during free speech leads to more uncommon associations when psychotic symptoms are present, resulting in incoherent speech (Elvevag et al., 2007). This feature can be objectively measured using a mathematical strategy known as latent semantic analysis (LSA; Landauer $\&$ Dumais, 1997), which estimates semantic proximity based on the cooccurrence of words within large, representative language corpora. By representing words as vectors in a high dimensional semantic space, it is possible to measure the semantic distance between words or groups of words. This approach was first used by a joint European-North American research team, and then by a Uruguayan team, to demonstrate that patients with schizophrenia speak with greater semantic inconsistency than control subjects (Cabana et al., 2011; Elvevag et al., 2007). More recently, within a youth population at risk for psychosis, a study with a major contribution from Argentinian and Brazilian researchers (our group) showed that it was possible to predict with $100 \%$ accuracy which subjects would eventually display actual psychotic episodes, based on quantitative features of quarterly clinical interviews recorded for up to 2.5 years (Bedi et al., 2015). The features employed for this prodromal investigation were the semantic inconsistency between consecutive sentences, maximum phrase length, and the amount of determiners (e.g., which). Altogether, these results point to a feasible way to track and prevent the onset of psychotic crises, even before the occurrence of a first episode during adolescence or early adulthood. This could give families a better chance to prevent major cognitive damages.

Early differential diagnosis with correct prognosis is also crucial to mitigate cognitive damage in psychotic patients. Especially for early onset, schizophrenia tends to produce more cognitive damage than bipolar mood disorder (Kaplan \& Sadock, 2009). Differential diagnosis is possible because thought disorders typical of patients with schizophrenia may differ substantially from those observed in patients with bipolar disorder (Andreasen \& Grove, 1986). In order to better characterize mental organization among psychotic patients, we developed a method based on graph theory to measure the complexity of the stream of thoughts as expressed by speech (Mota et al., 2012, 2014). When applied to Brazilian patients at mental institutions typical of Latin American public health settings, this method allowed the quantitative identification of bipolar disorder symptoms such as logorrhea and flight of ideas (Mota et al., 2012, 2014), as well as schizophrenia symptoms such as laconic talking, with a less connected and more linear structure, which altogether stand for the symptom 
known as poor speech (Mota et al., 2012, 2014). Measures of graph connectivity are significantly anticorrelated with negative symptoms (e.g., difficulty socializing and establishing ties with the interviewer), as well as cognitive symptoms (e.g., failure to understand abstract concepts) (Mota et al., 2014). This method can automatically distinguish schizophrenia, bipolar and control subjects with high accuracy (Mota et al., 2012, 2014). The distinction between these diagnostic entities leads to the identification of potential prognostic predictors, as indeed indicated by the fact that the expected course of schizophrenia, as compared to bipolar disorder, produces more severe cognitive impairment and hence a more difficult socialization. Having established this correlation, computational psychiatrists now need to carry on longitudinal studies in order to establish the predictive value of the graph-theoretical method for diagnosis, prognosis, and response to treatment in clinical situations such as prodrome or first episode. This will allow the early identification and treatment of the diseases that can lead to psychosis. It will also promote a deeper understanding of the distinct biological bases of schizophrenia and bipolar disorder, which have partially overlapping symptomatology but a quite different clinical course.

Another promising research line in computational psychiatry is related to the fact that speech features such as pitch and speed are very strongly affected by mood. In situations of euphoria, it is common to observe higher speech rate and higher voice amplitude, in comparison with times of sorrow. Voice samples can be collected on a daily basis with the help of a cell phone device, currently so ubiquitous, to generate a naturalistic, dense, and nonbiased speech sample of individuals diagnosed with bipolar disorder (Grunerbl et al., 2015). Prosodic measures of speech recorded by mobile applications have been shown to be useful in the identification of extreme mood episodes such as mania and depression (Grunerbl et al., 2015).

\section{Conclusions}

The early differential diagnosis of mental disorders affects the individual's life and epidemiological perspective and scaffolds the design of public policies for the prevention of mental distress. Interdisciplinary prevention leads to a mitigation of social impact, reduced risk factors, and improved welfare of the population. In Latin America, risk factors for mental illness are particularly prevalent, and there are few professionals effectively qualified to identify psychiatric vulnerabilities (Duarte et al., 2003). In this context, the use of automated methods for the objective quantification of prognostic predictors of mental health and cognition may greatly empower patients and psychiatrists as well, and it may help to break the mental disorder-poverty cycle that plagues the region. The fact that these computational methods for psychiatry have in large part been developed by Latin American researchers is an auspicious indication that the scientific gap 
between developed and developing countries may be decreasing in some fields.

\section{Acknowledgements}

This work was supported by Conselho Nacional de Desenvolvimento Científico e Tecnológico (CNPq), grants Universal 480053/2013-8 and Research Productivity 306604/2012-4 and 310712/2014-9; Coordenação de Aperfeiçoamento de Pessoal de Nível Superior (CAPES) Projeto ACERTA; Fundação de Amparo à Ciência e Tecnologia do Estado de Pernambuco (FACEPE); FAPESP Center for Neuromathematics (grant \# 2013/07699-0, S. Paulo Research Foundation FAPESP).

\section{References}

Adams, R. A., Huys, Q. J., \& Roiser, J. P. (2015). Computational sychiatry: Towards a mathematically informed understanding of mental illness. Journal of Neurology, Neurosurgery, and Psychiatry. doi: jnnp-2015-310737

Andreasen, N. C., \& Grove, W. M. (1986). Thought, language, and communication in schizophrenia: Diagnosis and prognosis. Schizophrenia Bulletin, 12(3), 348-359.

Baron-Cohen, S., Leslie, A. M., \& Frith, U. (1985). Does the autistic child have a "theory of mind"? Cognition, 21(1), 37-46. doi: 0010-0277(85)90022-8

Baxter, A. J., Patton, G., Scott, K. M., Degenhardt, L., \& Whiteford, H. A. (2013). Global epidemiology of mental disorders: What are we missing? PLoS One, 8(6), e65514. doi: 10.1371/journal.pone.0065514

Bedi, G., Carrillo, F., Cecchi, G. A., Slezak, D. F., Sigman, M., Mota, N. B., .. Corcoran, C. M. (2015). Automated analysis of free speech predicts psychosis onset in high-risk youths. npj Schizophrenia, 1, 15030. doi: 10.1038/npjschz.2015.30

Belfer, M. L., \& Rohde, L. A. (2005). Child and adolescent mental health in Latin America and the Caribbean: Problems, progress, and policy research. Revista Panamericana de Salud Pública, 18(4-5), 359-365. doi: S1020-49892005000900016

Bertola, L., Mota, N. B., Copelli, M., Rivero, T., Diniz, B. S., Romano-Silva, M. A., ... Malloy-Diniz, L. F. (2014). Graph analysis of verbal fluency test discriminate between patients with Alzheimer's disease, mild cognitive impairment and normal elderly controls. Frontiers in Aging Neuroscience, 6(185). doi: 10.3389/fnagi.2014.00185

Bleuler, E. (1911). Dementia praecox or the group of schizophrenias. New York: International Universities Press.

Brodersen, K. H., Deserno, L., Schlagenhauf, F, Lin, Z., Penny, W. D., Buhmann, J. M., $\&$ Stephan, K. E. (2014). Dissecting psychiatric spectrum disorders by generative embedding. NeuroImage: Clinical, 4, 98-111. doi: 10.1016/j.nicl.2013.11.002

Cabana, A., Valle-Lisboa, J. C., Elvevag, B., \& Mizraji, E. (2011). Detecting orderdisorder transitions in discourse: Implications for schizophrenia. Schizophrenia Research, 131, 157-164.

Canino, G., Shrout, P. E., Rubio-Stipec, M., Bird, H. R., Bravo, M., Ramirez, R., ... Martinez-Taboas, A. (2004). The DSM-IV rates of child and adolescent disorders in Puerto Rico: Prevalence, correlates, service use, and the effects of impairment. Archives of General Psychiatry, 61(1), 85-93. doi: 10.1001/archpsyc.61.1.85

Clark, D. B., Jones, B. L., Wood, D. S., \& Cornelius, J. R. (2006). Substance use disorder trajectory classes: Diachronic integration of onset age, severity, and course. Addictive Behaviors, 31(6), 995-1009. doi: S0306-4603(06)00086-4 
de la Barra, F. M. (2009). Epidemiología de trastornos psiquiátricos en niños y adolescentes: Estudios de prevalencia [Epidemiology of psychiatric disorders in children and adolescentes: Prevalence studies]. Revista Chilena de Neuro-Psiquiatría, 47(4), 303314.

de la Barra, M. F., Vicente, P. B., Saldivia, B. S., \& Melipillán, A. R. (2012). Estudio de epidemiología psiquiátrica en niños y adolescentes en Chile. Estado actual [Investigation of psychiatric epidemiology among children and adolescents in Chile]. Revista Médica Clínica Las Condes, 23(5), 521-529.

Díaz, J.-L. (2013). A narrative method for consciousness research.Frontiers in Human Neuroscience, 7(739), 1-12.

Dohrenwend, B. P., Levav, I., Shrout, P. E., Schwartz, S., Naveh, G., Link, B. G., ... Stueve, A. (1992). Socioeconomic status and psychiatric disorders: The causationselection issue. Science, 255(5047), 946-952.

Duarte, C., Hoven, C., Berganza, C., Bordin, I., Bird, H., \& Miranda, C. T. (2003). Child mental health in Latin America: Present and future epidemiologic research. International Journal of Psychiatry in Medicine, 33(3), 203-222.

Elvevag, B., Foltz, P. W., Weinberger, D. R., \& Goldberg, T. E. (2007). Quantifying incoherence in speech: An automated methodology and novel application to schizophrenia. Schizophrenia Research, 93(1-3), 304-316. doi: S0920-9964(07)00117-X

First, M. H., Spitzer, R. L., Gibbon, M., \& Williams, J. (1990). Structured clinical interview for DSM-IV Axis I disorders-Research version, patient edition (SCID-I/P). New York: New York State Psychiatric Institute.

Fleitlich, B. W., \& Goodman, R. (2000). Epidemiologia. Revista Brasileira de Psiquiatria, 22(Suppl. 2), 2-6.

Ford, T., Goodman, R., \& Meltzer, H. (2003). The British child and adolescent mental health survey 1999: The prevalence of DSM-IV disorders. Journal of the American Academy of Child and Adolescent Psychiatry, 42(10), 1203-1211.

Frith, U. (1997). The neurocognitive basis of autism. Trends in Cognitive Sciences, 1(2), 73-77. doi: 10.1016/S1364-6613(97)01010-3

Giel, R., de Arango, M. V., Climent, C. E., Harding, T. W., Ibrahim, H. H., LadridoIgnacio, L., . . Younis, Y. O. (1981). Childhood mental disorders in primary health care: Results of observations in four developing countries. A report from the WHO collaborative Study on Strategies for Extending Mental Health Care. Pediatrics, 68(5), 677-683.

Grunerbl, A., Muaremi, A., Osmani, V., Bahle, G., Ohler, S., Troster, G., . . L Lukowicz, P. (2015). Smartphone-based recognition of states and state changes in bipolar disorder patients. IEEE Journal of Biomedical and Health Informatics, 19(1), 140-148. doi: 10.1109/JBHI.2014.2343154

Insel, T. (2014). The NIMH Research Domain Criteria (RDoC) Project: Precision medicine for psychiatry. American Journal of Psychiatry, 171(4), 395-397. doi: 10.1176/appi.ajp.2014.14020138

Insel, T., Cuthbert, B., Garvey, M., Heinssen, R., Pine, D. S., Quinn, K., . . Wang, P. (2010). Research domain criteria (RDoC): Toward a new classification framework for research on mental disorders. American Journal of Psychiatry, 167(7), 748-751. doi: 10.1176/appi.ajp.2010.09091379

Kaplan, H. I., \& Sadock, B. J. (2009). Kaplan \& Sadock's comprehensive textbook of psychiatry. Baltimore, MD: Wolters Kluwer, Lippincott Williams \& Wilkins.

Kaufman, J., Gelernter, J., Hudziak, J. J., Tyrka, A. R., \& Coplan, J. D. (2015). The Research Domain Criteria (RDoC) Project and studies of risk and resilience in maltreated children. Journal of the American Academy of Child and Adolescent Psychiatry, 54(8), 617-625. doi: 10.1016/j.jaac.2015.06.001

Kessler, R. C., Amminger, G. P., Aguilar-Gaxiola, S., Alonso, J., Lee, S., \& Ustun, T. B. (2007). Age of onset of mental disorders: A review of recent literature. Current Opinion in Psychiatry, 20(4), 359-364. doi: 10.1097/YCO.0b013e32816ebc8c 
Kessler, R. C., Keller, M. B., \& Wittchen, H. U. (2001). The epidemiology of generalized anxiety disorder. Psychiatric Clinics of North America, 24(1), 19-39.

Kraepelin, E. (1906). Über sprachstörungen im traume [About language disorders in dreams]. Leipzig, Germany: Engelmann.

Krystal, J. H., \& State, M. W. (2014). Psychiatric disorders: Diagnosis to therapy. Cell, 157(1), 201-214. doi: 10.1016/j.cell.2014.02.042

Landauer, T. K., \& Dumais, S. T. (1997). A solution to Plato's problem: The Latent Semantic Analysis theory of the acquisition, induction, and representation of knowledge. Psychological Review, 104, 211-240.

Mercadante, M. T., Evans-Lacko, S., \& Paula, C. S. (2009). Perspectives of intellectual disability in Latin American countries: Epidemiology, policy, and services for children and adults. Current Opinion in Psychiatry, 22(5), 469-474. doi: 10.1097/YCO.0b013e32832eb8c6

Misra, V. (2014). The social brain network and autism. Annals of Neuroscience, 21(2), 69-73. doi: 10.5214/ans.0972.7531.210208

Montague, P. R., Dolan, R. J., Friston, K. J., \& Dayan, P. (2012). Computational psychiatry. Trends in Cognitive Sciences, 16(1), 72-80. doi: 10.1016/j.tics.2011.11.018

Moskowitz, A., \& Heim, G. (2011). Eugen Bleuler's Dementia praecox or the group of schizophrenias (1911): A centenary appreciation and reconsideration. Schizophrenia Bulletin, 37(3), 471-479. doi: 10.1093/schbul/sbr016

Mota, N. B., Furtado, R., Maia, P. P., Copelli, M., \& Ribeiro, S. (2014). Graph analysis of dream reports is especially informative about psychosis. Scientific Reports, 4, 3691. doi: 10.1038/srep03691

Mota, N. B., Vasconcelos, N. A., Lemos, N., Pieretti, A. C., Kinouchi, O., Cecchi, G. A., ... Ribeiro, S. (2012). Speech graphs provide a quantitative measure of thought disorder in psychosis. PLoS One, 7(4), e34928. doi: 10.1371/journal.pone.0034928

Nierenberg, A. A., Quitkin, F. M., Kremer, C., Keller, M. B., \& Thase, M. E. (2004). Placebo-controlled continuation treatment with mirtazapine: Acute pattern of response predicts relapse. Neuropsychopharmacology, 29(5), 1012-1018. doi: 10.1038/sj.npp.1300405

Oschilewsky, R. C., Gomez, C. M., \& Belfort, E. (2010). Child psychiatry and mental health in Latin America. International Review of Psychiatry, 22(4), 355-362. doi: $10.3109 / 09540261.2010 .503692$

Roberts, R. E., Attkisson, C. C., \& Rosenblatt, A. (1998). Prevalence of psychopathology among children and adolescents. American Journal of Psychiatry, 155, 715725.

Robins, L. N., \& Price, R. K. (1991). Adult disorders predicted by childhood conduct problems: Results from the NIMH Epidemiologic Catchment Area project. Psychiatry, 54(2), 116-132.

Rohde, L. A., Celia, S., \& Berganza, C. (2004). Systems of care in South America. In H. Remschmidt, M. Belfer, \& I. Gooyer (Eds.), Facilitating pathways, care, treatment and prevention in child and adolescent mental health (pp. 42-51). Heidelberg, Germany: Springer Verlag.

Wang, X. J., \& Krystal, J. H. (2014). Computational psychiatry. Neuron, 84(3), 638-654. doi: 10.1016/j.neuron.2014.10.018

Westeyn, T. L., Abowd, G. D., Starner, T. E., Johnson, J. M., Presti, P. W., \& Weaver, K. A. (2012). Monitoring children's developmental progress using augmented toys and activity recognition. Personal and Ubiquitous Computing, 16, 169-191. doi: 10.1007/s00779-011-0386-0

Yoshida, W., Dziobek, I., Kliemann, D., Heekeren, H. R., Friston, K. J., \& Dolan, R. J. (2010). Cooperation and heterogeneity of the autistic mind. Journal of Neuroscience, 30(26), 8815-8818. doi: 10.1523/JNEUROSCI.0400-10.2010 
NATÁLIA BEZERRA MOta is a PhD student at the Brain Institute, Federal University of Rio Grande do Norte. She completed a psychiatry residence and received a MSc in neuroscience from the Federal University of Rio Grande do Norte.

Mauro Copelli is associate professor of physics at the Physics Department, Federal University of Pernambuco. He received a PhD in physics from Limburgs Universitair Centrum.

SIDARTA RIBEIRO is full professor of neuroscience at the Brain Institute, Federal University of Rio Grande do Norte. He received a PhD in animal behavior from the Rockefeller University. 